\title{
Towards the optimization of photoacoustic sensors
}

\author{
B. Kost ${ }^{1}$, B. Baumann ${ }^{1}$, M. Wolff ${ }^{1,2} \&$ H. Groninga ${ }^{2}$ \\ ${ }^{1}$ Department of Mechanical and Production Engineering \\ Hamburg University of Applied Sciences, Germany \\ ${ }^{2}$ PAS-Tech GmbH, Zarrentin, Germany
}

\begin{abstract}
The detection sensitivity of photoacoustic sensors strongly depends on the design of the acoustical cell. It can be considerably improved by taking advantage of cell resonances, i.e., the acoustical eigenmodes of the measuring chamber. In order to optimize a photoacoustic system, it is key to understand precisely the influence of optical excitation, sound wave generation, cell shape and microphone detection on the signal strength in the sample cell. In this paper, first steps towards the optimization of photoacoustic cells are presented. The evaluation of the objective function, the signal strength, is based on a finite element analysis of the pressure field. As a start, independent optimization of some key parameters are investigated.
\end{abstract}

\section{Introduction}

Photoacoustic spectroscopy finds many applications in the field of concentration measurements of gaseous compounds, e. g. for medical examinations (breath tests), monitoring of emissions and immission control.

The photoacoustic effect is based on resonant absorption of light by specified molecules in a gas sample and the transfer of the excitation energy into thermal energy via inelastic collisions of the gas molecules. A modulated irradiation of the sample by a laser beam causes periodic pressure variations that can be detected by a microphone and measured using lock-in technique [1].

The most frequently used type of photoacoustic sensors is based on a cylinder shaped container [2]. In recent years the interest in photoacoustic cells with unconventional shapes is increasing. A so called T-cell, consisting of two intersecting cylinders, has been proposed in [3]. The optical absorption cylinder and the centri- 
cally perpendicularly mounted acoustical resonance cylinder form a T-geometry. At the end of the resonance cylinder the microphone is mounted. This design allows independent optimization of the key parameters affecting the signal strength. Figure 1 shows the experimental set-up, details can be found in [3]. X-cells show a similar function principle, only the resonance cylinder is split up in two parts. The geometry of both cell types can be seen in Figure 2. Table 1 lists the cell dimensions used in the following investigations. In [3] a T-cell with a diminution has been investigated experimentally and numerically. In the present paper the diminution has not been included in the model.

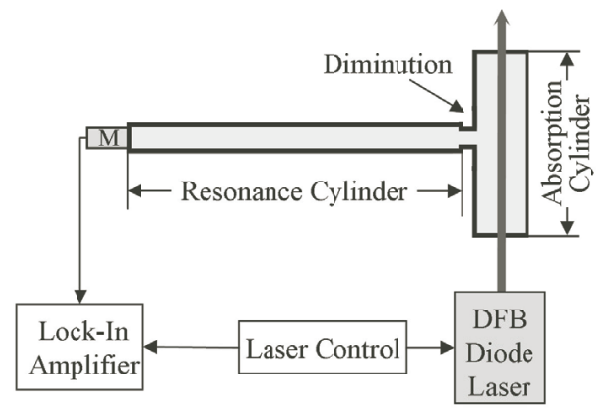

Figure 1: Experimental set-up of a photoacoustic sensor with T-cell.

Table 1: Dimensions of the photoacoustic T-cell and X-cell.

\begin{tabular}{lll}
\hline \hline Absorption Cylinder & Diameter & $D_{\mathrm{A}}=26 \mathrm{~mm}$ \\
& Length & $L_{\mathrm{A}}=82 \mathrm{~mm}$ \\
Resonance Cylinder & Diameter & $D_{\mathrm{R}}=11 \mathrm{~mm}$ \\
& Length & $L_{\mathrm{R}}$ adjustable \\
\hline \hline
\end{tabular}

\section{Analysis of photoacoustic sensors}

Particularly in the context of acoustically resonant trace gas detection, the eigenmodes of photoacoustic cells are of importance. The problem can be solved analytically for cells of cylindrical shape. Analytical solutions are naturally restricted to simple domain geometries and boundary conditions. Therefore, they are of limited use in "real-world" engineering applications. Numerical approximation techniques, like the finite element method, allow the handling of these complex problems. The consecutive calculations were performed using the finite element tool COMSOL Multiphysics 3.2 [4]. The results were obtained with the default solver parameters (tetrahedrons, quadratic Lagrange elements). Typical finite element meshes for T-cells and X-cells can be seen in Figure 2. 

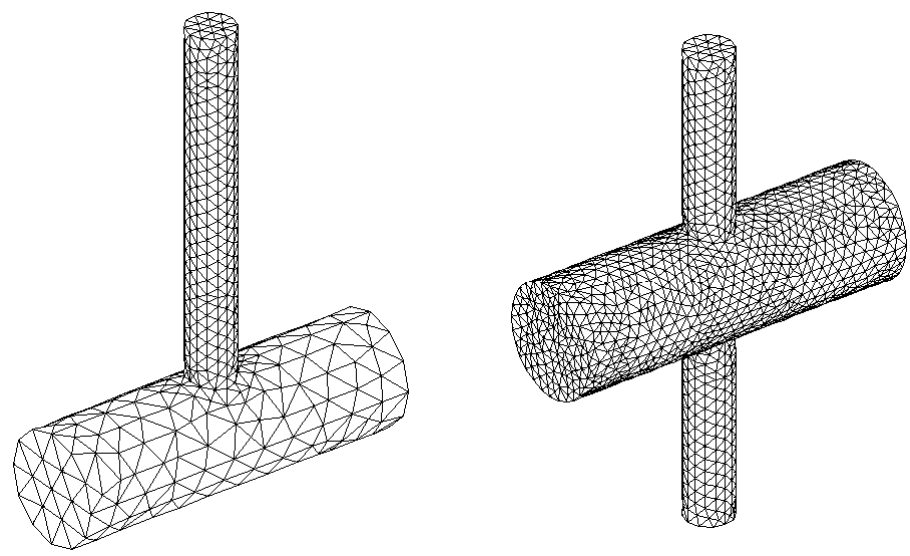

Figure 2: Typical finite element meshes of a T-cell (left) and a X-cell (right).

The formulation of the objective function in section 3 rely on a pressure value at a certain point in the photoacoustic cell. The calculation of the pressure field has been performed in the following manner:

- In a first step the eigenvalue problem has been examined, i. e., the homogeneous wave equation has been solved numerically assuming the cell walls sound hard. Details of this access can be found in $[5,6]$. One non-trivial eigenmode of a T-cell with short resonance cylinder is depicted in Figure 3. For the following steps the first eight eigenmodes have been taken into account.

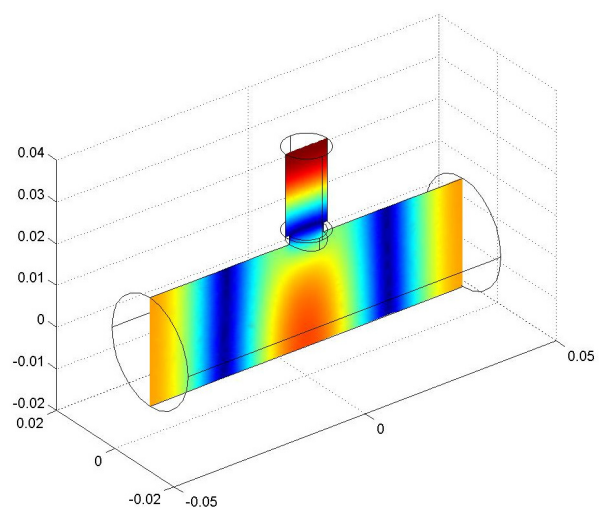

Figure 3: Non-trivial eigenmode of a T-cell with short resonance cylinder. Depicted is the absolute value of the pressure field $|p|$. 
- It is well known that the solution of the inhomogeneous wave equation can be expressed as a superposition of the acoustical modes. Therefore, the eigenmode structure of the preceding step, i.e., the modes and associated eigenfrequencies, is the basis for an expansion of the pressure field into acoustical eigenmodes.

- The inhomogeneous wave equation does not contain terms that account for losses. Loss effects are included via the introduction of quality factors in the amplitudes [7].

A detailed description of this approach and a comparison with experimental data can be found in $[8,9]$. These publications contain the associated gas parameters as well.

\section{Optimization of photoacoustic sensors}

The standard model of a nonlinear optimization problem is given by an objective function in $n$ design variables

$$
f: \mathbb{R}^{n} \longrightarrow \mathbb{R}, \quad \boldsymbol{x} \longrightarrow f(\boldsymbol{x})=: F
$$

and $m$ and $l$ constraints functions

$$
\begin{aligned}
& g_{i}: \mathbb{R}^{n} \longrightarrow \mathbb{R}, \quad i=1,2, \ldots, m \\
& h_{j}: \mathbb{R}^{n} \longrightarrow \mathbb{R}, \quad j=1,2, \ldots, l .
\end{aligned}
$$

With the inequality constraints $g_{i}$ and equality constraints $h_{j}$ the subset $\mathbf{X} \subset \mathbb{R}^{n}$ defined by

$$
\mathbf{X}:=\left\{\boldsymbol{x} \in \mathbb{R}^{n} \mid g_{i}(\boldsymbol{x}) \leq 0, i=1,2, \ldots, m ; h_{j}(\boldsymbol{x})=0, j=1,2, \ldots, l\right\}
$$

is called the feasible region. A solution of the nonlinear maximization problem can be expressed by

$$
\boldsymbol{x}^{*}:=\operatorname{argmax}\{f(\boldsymbol{x}) \mid \boldsymbol{x} \in \mathbf{X}\} .
$$

With respect to the optimization of photoacoustic sensors a lot of different optimization models can be formulated. In the following the objective function is set to $f:=p_{M}^{2}$. The function $p_{M}$ determines the value of the pressure field at the microphone position which is a measure of the signal strength of the photoacoustic cell. According to this, the objective function value $F$ can be calculated by a finite element analysis as described in section 2 .

The efficiency of a photoacoustic sensor depends on a variety of influences. System variables (optimization parameters) of a photoacoustic cell are e.g. geometrical dimensions, shape parameters, the location of the laser beam, the location of the resonance cylinder and the number and locations of microphones.

Restrictions on the design could be given e. g. by geometrical constraints due to design or handling requirements, by a limitation of the cell volume (for medical purposes maybe breath or device volume), a predescribed frequency band 
to eliminate disturbances due to environmental conditions and last but not least the parameters could be restricted simply by lower and upper bounds due to technical reasons.

In the following some preliminary investigations with respect to the optimization of photoacoustic cells are shown.

\subsection{Optimization of laser beam location for a T-cell}

Depending on the laser beam location different eigenmodes can be excitated. The task is to find the laser position and direction which leads to the strongest signal.

\subsubsection{Laser beam coordinates}

In a first step the laser beam is assumed to be parallel to the $z$-axis and the influence of the laser beam position on the signal strength has been investigated. For this purpose the cross-sectional area has been covered by a regular grid $\left(x_{i}, y_{i}\right)$ of evaluation points (see Figure 4 for the coordinate system of a T-cell). The grid points have to fulfill the inequality

$$
g(x, y)=x^{2}+y^{2}-r_{L}^{2} \leq 0
$$

where $r_{L}=\left(D_{A} / 2\right)-r_{B}$ is a given limit for the radius and $r_{B}=2 \mathrm{~mm}$ is approximately the laser beam radius. This constraint has been imposed to ensure a proper energy input into the system.

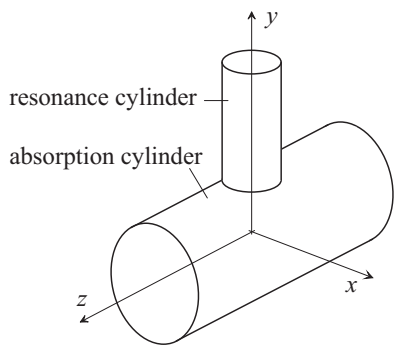

Figure 4: Coordinate system of T-cell. In the following the use of "top", "bottom", "horizontal" etc. refers to this system.

In Figure 5 the objective function is shown for different length $L_{R}$ of the resonance cylinder. Depending on the length $L_{R}$ the objective function in some cases is not concave. The strongest influence of the laser beam position occurs for $L_{R}=1 \mathrm{~cm}$. For larger resonance cylinder lengths the objective function tends to be uniform. In all cases the maximum value $F_{\max }$ on the objective function surfaces can be located at the position $\left(0, y_{\text {min }}\right)$ which suggests that a laser beam going through the lower part of the absorption cylinder results in the best signal. 
$L_{R}=1 \mathrm{~cm}$

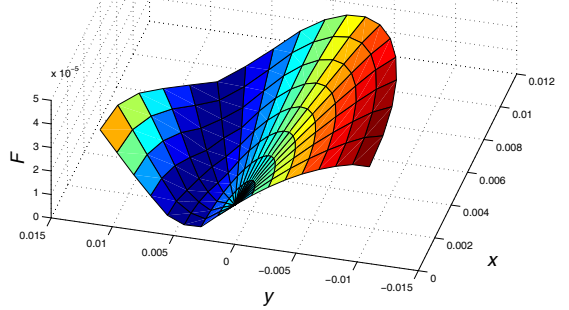

$L_{R}=4 \mathrm{~cm}$

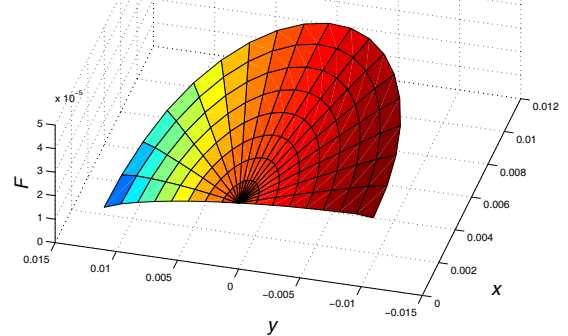

$L_{R}=2 \mathrm{~cm}$

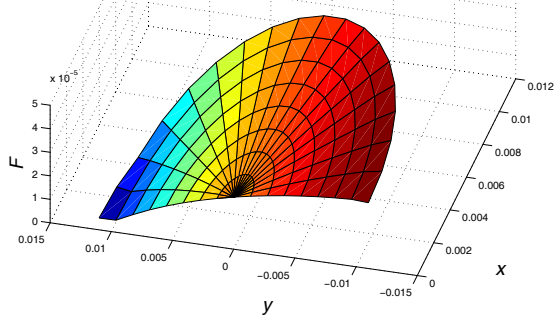

$L_{R}=8 \mathrm{~cm}$

Figure 5: Objective function of T-cells with different resonance cylinder length $L_{R}$ for the laser beam position problem.

In Figure 6 the extreme values, $F_{\max }$ and $F_{\min }$, of the objective function and the value $F_{\text {ori }}$ for the coordinate system origin have been depicted for different resonance cylinder length. The difference $F_{\max }-F_{\min }$ can be interpreted as a measure for the influence of the laser position on the signal strength. Considering this difference the tendency of the objective function to become uniform with larger resonance cylinder lengths is clearly visible. For $L_{R}>6 \mathrm{~cm} F_{\text {ori }} \approx F_{\max }$ and this indicates that a laser beam going through the central position $(0,0)$ could be sufficient to obtain approximately the maximal objective function value.

The most important information of Figure 6 is the fact that a strong signal strength can be achieved with a small resonance cylinder length in the range of $L_{R}=1 \mathrm{~cm}$. The maximum for a resonance cylinder length of $1 \mathrm{~cm}$ is approximately the same as for $L_{R}=16 \mathrm{~cm}$ (not shown in the figure). This offers the opportunity to build small and compact T-cell sensors. But in this context it has to be taken into account that the sharp peak in the graph displays the sensitivity against length variations.

\subsubsection{Laser beam inclination}

Inspection of the data basis of Figure 5 reveals that the sensitivity of the objective function in $x$-direction is nearly zero independent from the resonance cylinder 


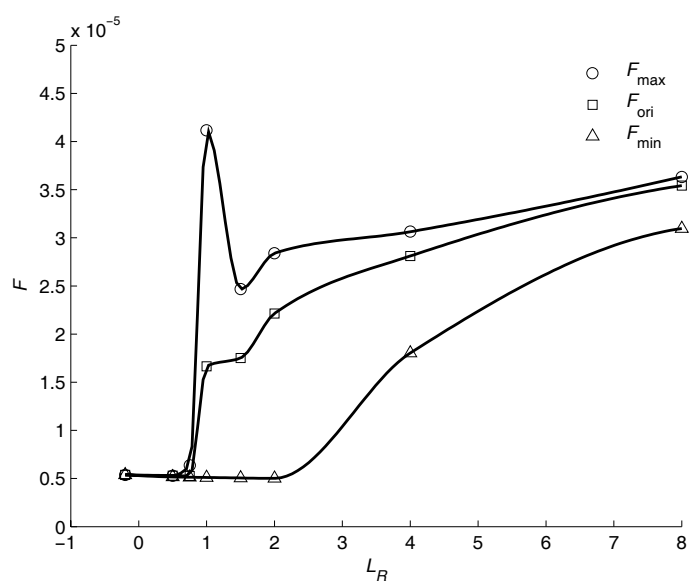

Figure 6: Comparison of extreme values of the objective function for a T-cell with different resonance cylinder length $L_{R}$.

length. This is the reason why in the following investigation a potential dependency on the lateral direction has been omitted.

To model the laser beam inclination a set of points $\left(0, y_{e_{i}},-\left(L_{A} / 2\right)\right)$ in the entry cross-sectional area and $\left(0, y_{o_{i}},\left(L_{A} / 2\right)\right)$ in the outlet cross-sectional area have been defined regarding $\left|y_{e_{i}}\right|-r_{L} \leq 0$ and $\left|y_{o_{i}}\right|-r_{L} \leq 0$. The difference $y_{o_{i}}-y_{e_{i}}$ is a measure for the inclination of the laser beam.

The objective function value for the combinations $\left(y_{e_{i}}, y_{o_{i}}\right)$ is displayed in Figure 7. As was expected after the investigation of section 3.1.1 the influence of the resonance cylinder length is similar. The objective function can be not concave for very short and is nearly uniform for longer cylinders. The best result can be achieved with $y_{e}=y_{o}$ in the lower part of the absorption cylinder. This is a laser beam going parallel to the $z$-axis.
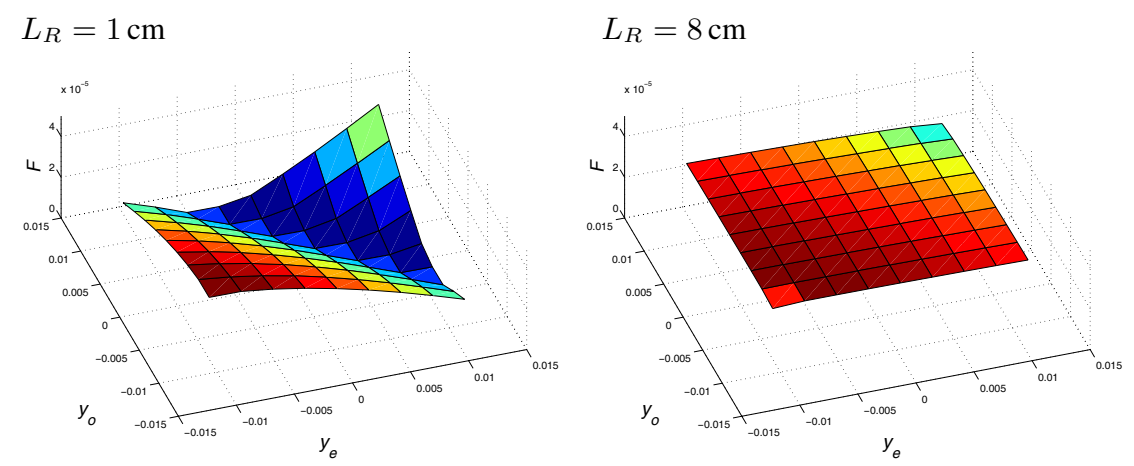

Figure 7: Objective function of T-cells with different resonance cylinder length $L_{R}$ for the laser beam inclination problem. 


\subsection{Optimization of cell geometry}

\subsubsection{Vertical location of the resonance cylinder between T-cell and X-cell}

For the investigation in this section the laser beam has been held fix, going in $z$-direction through the center of the absorption cylinder.

Starting from a T-cell configuration with a resonance cylinder on top of the absorption cylinder (see Figure 2, left), the resonance cylinder has been shifted step by step in the negative $y$-direction. The shift has been performed in such a way that the total length $L_{R}$ of the resonance cylinder has been split up in two parts located at the top and at the bottom of the absorption cylinder. A shift value of $y_{s}=$ $\left(L_{R} / 2\right)$ refers to the symmetrical situation, i. e., the X-cell (see Figure 2, right) and $y_{s}=L_{R}$ is a T-cell with resonance cylinder at the bottom of the absorption cylinder. The microphone is mounted on top of the upper part of the two resonance cylinders, in case of $y_{s}=L_{R}$ that means on the surface of the absorption cylinder. No constraints have been considered in this investigation. The results can be seen in Figure 8. For all investigated parameter values $L_{R}$ the T-cell with resonance cylinder on top of the absorption cylinder gives the strongest signal strength.

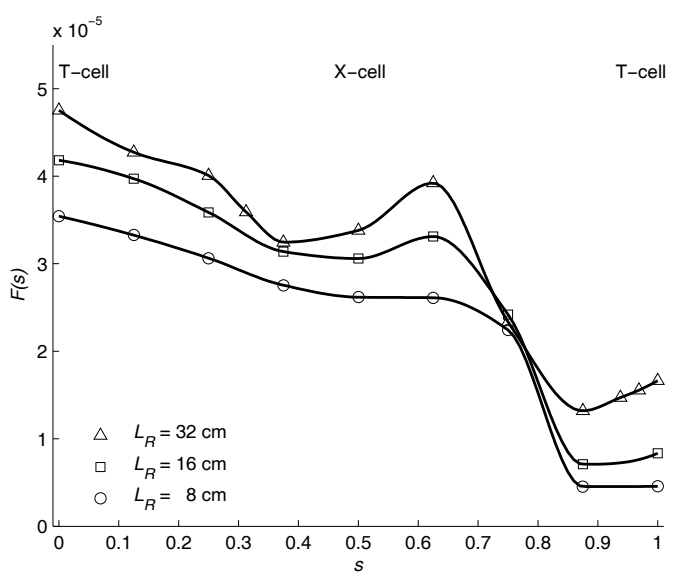

Figure 8: Objective function values for unconventional cell shapes between T-cell and $\mathrm{X}$-cell with different total resonance cylinder length $L_{R}$. The abszissa is $s=\left(y_{s} / L_{R}\right) . s=0$ refers to a T-cell with a resonance cylinder on top, $s=1$ with a single resonance cylinder at the bottom of the absorption cylinder.

A closer examination of Figure 8 leads to a suggestion with respect to the design of the photoacoustic sensor. If two microphones will be used, one located at the top of the upper another at the bottom of the lower resonance cylinder part, the two signals could be combined. A possible difference of phase could be compensated electronically so that the in-phase signals are considered only. Figure 9 shows an estimation of this effect. The objective function values $F(s)$ of Figure 8, where 
$s=\left(y_{s} / L_{R}\right)$ is the normalized shift, are processed in the following manner $F(s)+$ $F(1-s)$ to simulate the presence of two microphones. The positions of the maxima in Figure 9 show that the strongest signal can be achieved with asymmetrical cell types.

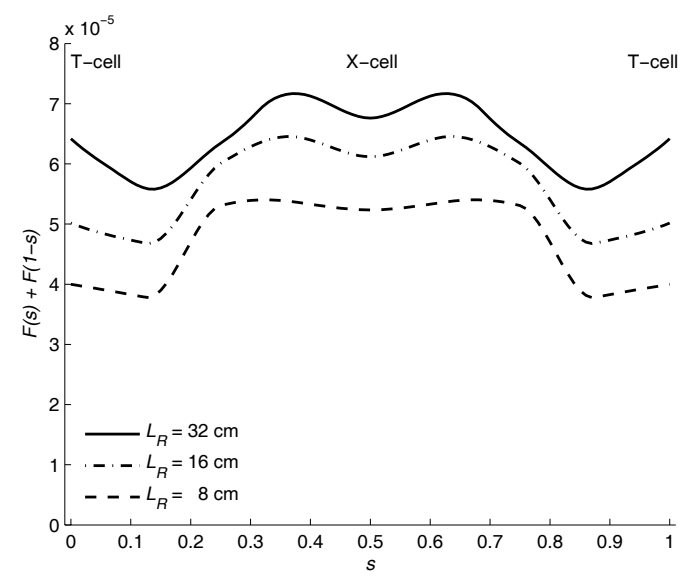

Figure 9: Simulated signal of two combined microphones.

\subsubsection{Shape optimization}

The investigation of this section has been carried out with a single cylinder in $z$-direction as a start configuration. The laser beam has been centrally oriented along the cylinder axis. The microphone is attached to the cylinder shell at one of the ends.

As a first step towards shape optimization of photoacoustic sensors the cylinder has been modelled in three parts. The four radii of these parts are the design parameters. Figure 10 depicts the shape and the pressure field after some optimization steps and shows the technical potential at hand.

Further investigations should include a more precise modelling of the basic geometry and constraints, e.g. a prescribed lower bound for the radii because of the laser beam width or an equality constraint due to a specified cell volume.

\section{Concluding remarks}

The aim of the paper is to investigate the feasibility of applying optimization methods to design photoacoustic sensors. On this account some basic configurations have been investigated.

With respect to T-cells the exploration of the objective function, the signal strength, shows that the laser position has a strong effect depending on the cell geometry, i. e., the length of the resonance cylinder. For small resonance cylinders 


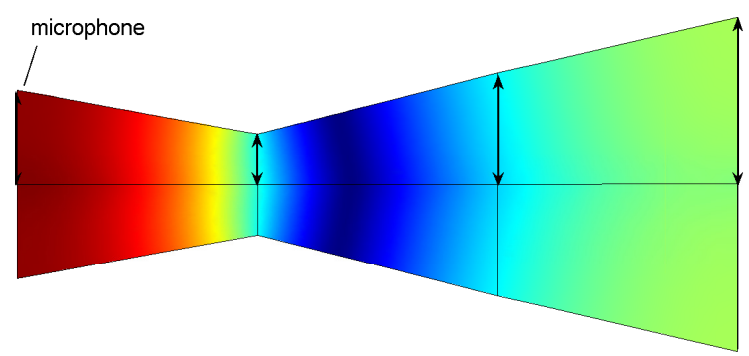

Figure 10: Optimized shape with pressure distribution. The four optimization variables are indicated by double arrows.

strong signals can be achieved provided the laser beam is no longer centrally oriented. It could also be shown that a laser beam inclination in the symmetry plane of a T-cell has no advantage over laser beam alignments parallel to the axis of the absorption cylinder.

Another investigation shows that it could be useful to build asymmetrical photoacoustical cells with two microphones at the end of the resonance cylinders to enhance the signal strength.

Furthermore, first results of a shape optimization of the photoacoustic cell are presented.

\section{References}

[1] Demtröder, W., Laser Spectroscopy. Springer-Verlag: Berlin, 2002.

[2] Michaelian, K.H., Photoacoustic Infrared Spectroscopy. Wiley-Interscience: Hoboken, 2003.

[3] Wolff, M., Groninga, H.G., Baumann, B., Kost, B. \& Harde, H., Resonance investigations using PAS and FEM. Acta Acustica, 91, pp. 1477-1481, Suppl 1, 99, 2005.

[4] COMSOL-Homepage: www.comsol.com.

[5] Baumann, B., Kost, B., Groninga, H. \& Wolff, M., Eigenmodes of photoacoustic T-cells. Proc. of the FEMLAB Conference 2005, Frankfurt a.M., pp. 231236, 2005.

[6] Baumann, B., Kost, B., Groninga, H. \& Wolff, M., Eigenmode analysis of photoacoustic sensors via finite element method. Review of Scientific Instruments, 77, 044901, 2006.

[7] Kreuzer, L.B., The physics of signal generation and detection. Optoacoustic Spectroscopy and Detection, ed. Y.H. Pao, Academic: London, pp. 1-25, 1977.

[8] Baumann, B., Wolff, M., Kost, B. \& Groninga, H., Calculation of quality factors and amplitudes of photoacoustic resonators. Proc. of the COMSOL Conference 2006, Frankfurt a.M., pp. 134-138, 2006.

[9] Baumann, B., Wolff, M., Kost, B. \& Groninga, H., Finite element calculation of photoacoustic signals. Applied Optics, accepted for publication. 